\title{
Supporting Information: Electron dynamics in a 2D nanobubble: A two-level system based on spatial density
}

\author{
Roberto Rosati, ${ }^{1}$ Frank Lengers, ${ }^{2}$ Christian Carmesin, ${ }^{3}$ Matthias Florian, ${ }^{3}$ \\ Tilmann Kuhn, ${ }^{2}$ Frank Jahnke, ${ }^{3}$ Michael Lorke, ${ }^{3}$ and Doris E. Reiter ${ }^{2}$ \\ ${ }^{1}$ Department of Physics, Philipps-Universität Marburg, Renthof 7, D-35032 Marburg, Germany* \\ ${ }^{2}$ Institut of Solid State Theory, University of Münster, 48149 Münster, Germany \\ ${ }^{3}$ Institute for Theoretical Physics, University of Bremen, P.O. Box 330440, 28334 Bremen, Germany
}

\section{SIMULATION DETAILS}

Our simulation is a multi-step process: (i) We perform a microscopic calculation of the nanobubble potential based on a combination of valence force field and 6-band tight-binding model. (ii) The nanobubble potential is inserted into the Schrödinger equation for K-valley electrons, yielding bound and free states together with the corresponding energies. (iii) Using the wave functions obtained from the Schrödinger equation as basis states, we set up the equation of motion for the electron density matrix in the framework of a Lindblad superoperator technique. (iv) As initial condition we construct wave packets in the subspace of the free states localized at a certain distance from the nanobubble and calculate their time evolution according to the density matrix equation. We here describe these steps in detail:

Regarding step (i): For the simulation of the nanobubble we use a combination of atomic force field calculations with tight binding simulations. Starting from a paraboloid profile of the nanobubble, the atoms in the upper TMDC layer are relaxed, while the atoms in the lower layer are kept fixed. This part of the simulation is performed using a REAX potential [1] with the parametrization from Ref. [2] within a valence force field calculation, which is capable of accurately describing bond deformations under strain as well as continuous bond formation and breaking dynamics. Based on these valence force field simulations, new equilibrium positions of the individual atoms in the bended material are determined. Information about the displaced atoms is used in a subsequent tight-binding electronic-state calculation for the supercell structure. For a supercell with an in-plane extension of $130 \mathrm{~nm}$ and up to $1.2 \times 10^{6}$ atoms, a 6-band tight-binding Hamiltonian is solved as discussed in Ref. [3]. Strain-induced local band gap changes arising from the displaced atomic positions are included via a generalized Harrison rule [4].

Additionally, when locally detaching the upper layer from the substrate underneath and changing from a commensurate bilayer to monolayer-like structures across the nanobubble, the upper layer is subject to a modified dielectric environment and electronic hybridization is hence different than in a bilayer [5-8]. As discussed in Ref. [3], these effects are included in the calculation by changing individual tight-binding parameters.

Regarding step (ii): We insert the potential evaluated in step (i) in a Schrödinger equation where we assume that the eigenstates for the bare-monolayer behave around the $\mathrm{K}$ valley as scalar plane waves with free eigenenergies $E_{\mathbf{k}}=\left(\lambda_{c}+\lambda_{v}\right) / 2+a_{0} t \sqrt{\left[\left(\Delta_{G}-\left(\lambda_{v}-\lambda_{c}\right)\right) /\left(2 a_{0} t\right)\right]^{2}+|\mathbf{k}|^{2}}[9]$, with $a_{0}=3.193 \AA, \Delta_{G}=1.66 \mathrm{eV}, t=1.1 \mathrm{eV}, \lambda_{v}=75$ $\mathrm{meV}$ and $\lambda_{c}=-1.5 \mathrm{meV}$ [10, 11], cf. also Ref. [9].

Regarding step (iii): We further set up the equations of motion taking into account the free evolution of the electronic wave packet (see point (iv) below for its initial definition) and scattering with LO phonons via Fröhlich interaction. While for excitons intervalley scattering can be very important [12-15], despite efficient Hamiltonian coefficients [16] free electrons close to the K-valley energy mininum cannot directly scatter in the free states of other valleys due to energy conservation or uneffectiveness of absorption of phonons with finite energies at cryogenic temperatures. In view of the picoseconds scattering times [17] and tens of picoseconds energy-thermalization time of related excitonic states [14], intravalley scattering with acoustic phonons of $\mathrm{K}$ electrons is expected to take place at longer timescales than the ones here considered, hence it is neglected. As a consequence we restrict ourselves to the free coherent evolution for the free distribution. The scattering between free and bound states is dominated by optical phonons modes due to energy separation between emitting and receiving states[18-20], as shown recently for excitons in Mo-based TMDC monolayers [21]. In particular here we focus on the effective scattering with LO phonon via Fröhlich interaction, whose scattering coefficients between electronic states in valley $\mathrm{K}$ with momenta $\mathbf{k}, \mathbf{k}+\mathbf{q}$ are given by $g_{\mathbf{q}} \equiv\left\langle\mathbf{k}+\mathbf{q}\left|\hat{H}_{e-L O}\right| \mathbf{k}\right\rangle$, where the electron-LO phonon Hamiltonian $H_{e-L O}$ follows Ref. [22]. These scattering coefficients have been inserted in a Lindblad approach for the carrier capture $[9,20]$, which is able to describe the scattering from the delocalized

*rosatir@uni-marburg.de 


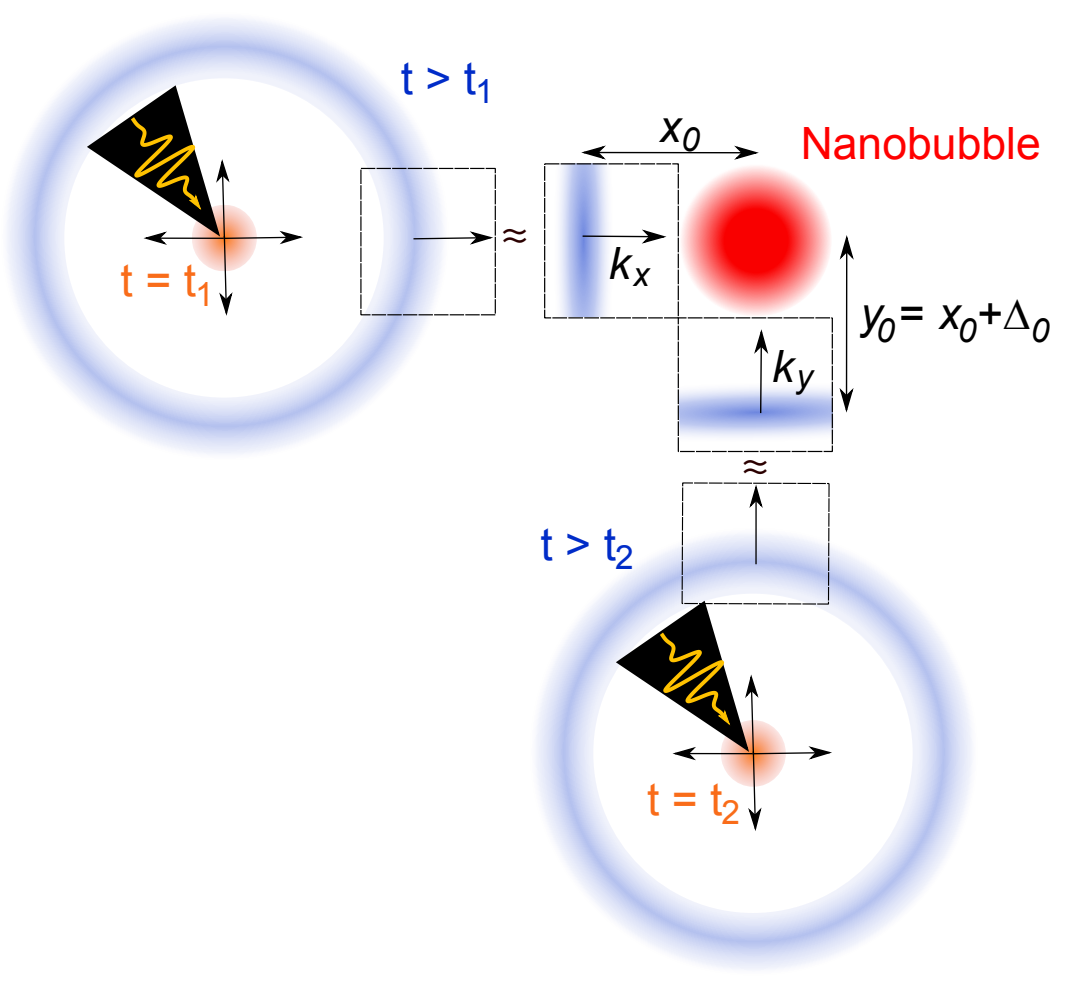

Fig. S 1. Possible experimental realization of the considered setup. Two strongly localized excitation sources lead to the considered wave fronts moving in $x$ and $y$ direction (marked parts of the ring-like wave packets). A temporal delay $t_{2}-t_{1}$ between the excitation of the two wave packets can be used to induce a difference in starting positions $\Delta_{0}=y_{0}-x_{0}$ as used in the simulations.

states into the localized ones not only tracking the energy of the emitting states, but also the spatial information. Key to describe the dynamics in this highly inhomogeneous case is the off-diagonal nature of the Lindblad superoperator together with an energetic broadening of the energy conservation, the latter previously included by comparison with the full quantum kinetics calculations [20], although more recent studies have shown similar broadenings [21].

Finally, regarding point (iv) we choose a wave-front type packet, which in the density matrix and in the basis of the free TMDC states and for the $x$-propagating case can be written as [9]

$$
\rho_{\mathbf{k}+\frac{\mathbf{k}^{\prime}}{2}, \mathbf{k}-\frac{\mathbf{k}^{\prime}}{2}} \propto e^{-\frac{1}{2}\left(k_{x}^{\prime} \Delta\right)^{2}} e^{-i k_{x}^{\prime} x_{0}} e^{-\left(\frac{\left(\hbar^{2} k_{x}^{2}\right) /\left(2 m^{*}\right)-E_{0}}{\sqrt{2} \Delta_{E}}\right)^{2}} \Theta\left(k_{x}\right) \delta\left(k_{y}^{\prime}\right) \delta\left(k_{y}\right)
$$

where $\mathbf{k} \equiv\left(k_{x}, k_{y}\right)$ is the $2 \mathrm{D}$ wave vector and $\Theta\left(k_{x}\right)$ is the Heaviside step function. The wave packet propagating along $y$ has the same form with $k_{x}^{\prime} \leftrightarrow k_{y}^{\prime}$ and $\left(x_{0} \leftrightarrow y_{0}\right)$. The wave packets have a finite width in space and energy of $\Delta=10 \mathrm{~nm}$ and $\Delta_{E}=5 \mathrm{meV}$, respectively, and are centered at $\mathbf{r}_{0} \equiv\left(x_{0}, 0\right)$ or $\left(0, y_{0}\right)$ for propagation along $x$ and $y$, respectively. The excess energy, which determines the velocity as well as the bound states which are energetically favorable for the carrier capture, is taken to be $E_{0}=22.5 \mathrm{meV}$, i.e., an energy close to $\left(E_{s}+E_{p}\right) / 2+\mathrm{E}_{L O}$, with $E_{s}$ and $E_{p}$ being the energy of state 1 and nearly degenerate states 2 and 3, respectively (see main manuscript). Such a wave packet can in principle be found in certain distances from the excitation spot of a strongly localized near-field source [9]. The energetic width $\Delta_{E}=5 \mathrm{meV}$ corresponds then to an ultrafast excitation of about $150 \mathrm{fs}$ duration. These length and timescales could be combined as typically done in the field of ultrafast nano-optics (see, e.g., [23] for a review on experimental applications). These experimental developments have led to extensive studies of nanometric wave packets (see, e.g., [24-26]). A specific setup for the presented system is given in Fig. S1, where two near-field sources lead to the considered wave packets in $x$ and $y$ direction impinging on the nanobubble. In this setup a temporal delay $t_{2}-t_{1}$ between the exciting pulses can be used to effectively tune the difference in starting positions $\Delta_{0}$.

Note that we work in the single-band approximation with the standard single-particle dispersion [10, 11], while also excitonic effects in TMDC monolayers are very prominent $[27,28]$. Nevertheless beside the formation of deeply-bound excitons [29, 30], in competition with spatial separation between electrons and holes [31], we also expect electronic wave packets able to travel through the monolayer and then be captured in the here-considered ultrafast timescale. 


\section{OCCUPATIONS AND COHERENCES OF THE CAPTURED ELECTRONS}

While in the manuscript we focus purely on the description using the real-valued density, it is interesting to consider the occupation and the coherences of the bound states. More details on the quantum mechanical description of the states using the density matrix formalism can be found in previous publications $[9,18-20,32,33]$.

In the density matrix formalism the single particle density matrix within the eigenbasis states $|i\rangle$ is given by

$$
\hat{\rho} \equiv \sum_{i, j} \rho_{i j}(t)|i\rangle\langle j|
$$

from which the spatial distribution is calculated via

$$
n(\mathbf{r}, t) \equiv \sum_{i, j} n_{i j}(\mathbf{r}, t) \quad \text { with } n_{i j}(\mathbf{r}, t)=\rho_{i j}(t) \Psi_{i}(\mathbf{r}) \Psi_{j}^{*}(\mathbf{r}) .
$$

In our case the states $i=1, \ldots 5$ correspond to the bound states in the nanobubble potential (see discussion in the main text). Since for our initial conditions the the occupation of the states $\Psi_{4}$ and $\Psi_{5}$ is negligible, we will restrict our discussion to states $i=1,2,3$.

The occupations and coherences of the states are then given respectively by

$$
f_{i}=\rho_{i i}=\langle i|\hat{\rho}| i\rangle \quad \text { and } \quad p_{i j}=\rho_{i j, i \neq j}=\langle i|\hat{\rho}| j\rangle_{i \neq j} .
$$

The most remarkable feature is that the capture from the 2D system into the 0D localized potential results not only in occupations, but in a superposition state of the different states [9]. The general density matrix reads

$$
\hat{\rho}=\frac{1}{2} \sum_{i=1}^{3} f_{i}|i\rangle\left\langle i\left|+e^{-i \omega t} \sum_{i=2}^{3} \tilde{p}_{1 i}\right| 1\right\rangle\langle i|+\text { H.c. },
$$

with $\omega=\left(E_{s}-E_{p}\right) / \hbar$ and H.c. denoting the Hermitian conjugate, and where we introduced $p_{1 i}=\tilde{p}_{1 i} \exp (-i \omega t)$ in the interaction picture. This equation already shows that the capture into the superposition state results in a dynamics of the captured density with the frequency $\omega$. The shape of the dynamics, however, is determined by the magnitude of the occupations $f_{i}$ together with the absolute values of the coherences $p_{12}$ and $p_{13}$ and most importantly the relative time difference between the coherences, which in turn is determined by the time of the carrier capture.

One example of carrier capture dynamics is shown in Fig. S2, where the dynamics of (a) the occupations and (b) the coherences is shown. We stress again that the capture happens locally, i.e., when the wave packet is close to the nanobubble as can be seen in the corresponding finite rise time of the occupations [20]. The final capture is slightly stronger into state 1 then into the excited states 2 and 3 as seen in Fig. S2(a), while the coherences after the capture in Fig. S2(b) are equally strong. However, the build-up of the population and in particular the coherences is different: The wave packet traveling along the $x$-direction induces the coherence $p_{12}$, while the wave packet traveling along $y$-direction induces $p_{13}$. When the latter wave packet is delayed, $p_{13}$ builds up later, but the final value $\left|p_{13}(t \rightarrow \infty)\right|$ is almost independent of $\Delta_{0}$. One may therefore approximate the dynamics of $p_{13}$ as $p_{13} \approx\left|p_{13}(t \rightarrow \infty)\right| \Theta\left(t-t_{V}\right) \exp \left(-i \omega\left(t-t_{V}\right)\right)$ with the Heaviside function $\Theta$ when approximating the build-up of coherence as instantaneous at the arrival time $t_{V}=y_{0} / v$ of the wavepacket in $y$-direction. Accordingly we find for these three examples the diagonal movement, the circular movement and the anti-diagonal movement due to the phase difference $\omega\left(t_{H}-t_{V}\right)=\omega \Delta_{0} / v$.

From the microscopic picture given in Eq. (1) we obtain the spatial profiles of the oscillation of the density as defined in Eq. (2) of the main manuscript. In particular we find that $c_{V}=2\left|p_{13}(t \rightarrow \infty)\right| \psi_{1}(\mathbf{r}) \psi_{3}(\mathbf{r})$ and $t_{V}=y_{0} / v$ with the starting distance $y_{0}$ from the QD and replacing $3 \rightarrow 2$ we get similar expressions for $c_{H}$ and $t_{H}$. This connects the quantum mechanical picture of the states on the Poincaré sphere to the dynamics of the captured density. 

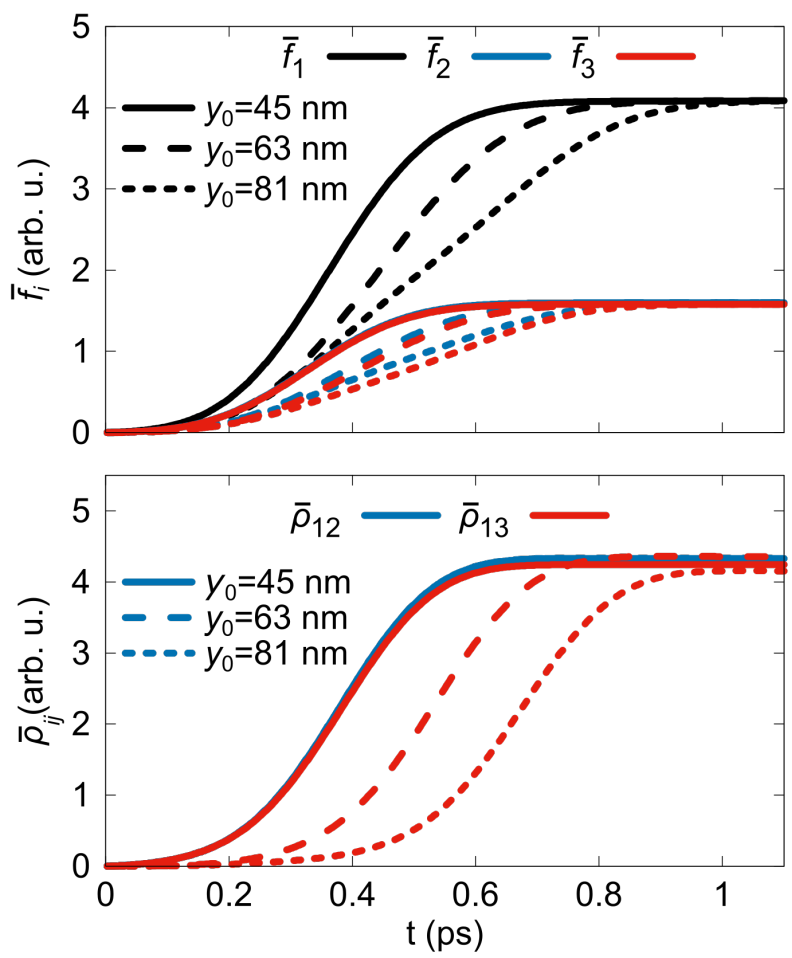

Fig. S 2. Dynamics of (a) the occupations of the bound states and (b) the absolute values between the coherences of the bound states for excitation with two wave packets from $x$ (initial distance from the nanobubble $x_{0}=45 \mathrm{~nm}$ ) and $y$-direction with different starting distances $\Delta_{0}=y_{0}-x_{0}=0,18$ and $36 \mathrm{~nm}$. These correspond to the dynamics shown in Fig. 3(middle, bottom row) and COM movements seen in Fig. 4(c), (e) and (d) of the main manuscript, respectively.

[1] A. C. T. van Duin, S. Dasgupta, F. Lorant, and W. A. Goddard, ReaxFF: a reactive force field for hydrocarbons, J. Phys. Chem. A 105, 9396 (2001).

[2] A. Ostadhossein, A. Rahnamoun, Y. Wang, P. Zhao, S. Zhang, V. H. Crespi, and A. C. T. van Duin, ReaxFF reactive force-field study of molybdenum disulfide $\left(\mathrm{MoS}_{2}\right)$, J. Phys. Chem. Lett. 8, 631 (2017).

[3] C. Carmesin, M. Lorke, M. Florian, D. Erben, A. Schulz, T. O. Wehling, and F. Jahnke, Quantum-dot-like states in molybdenum disulfide nanostructures due to the interplay of local surface wrinkling, strain, and dielectric confinement, Nano Lett. 19, 3182 (2019).

[4] S. Froyen and W. A. Harrison, Elementary prediction of linear combination of atomic orbitals matrix elements, Phys. Rev. B 20, 2420 (1979).

[5] T. Cheiwchanchamnangij and W. R. L. Lambrecht, Quasiparticle band structure calculation of monolayer, bilayer, and bulk $\mathrm{MoS}_{2}$, Phys. Rev. B 85, 205302 (2012).

[6] M. Rösner, C. Steinke, M. Lorke, C. Gies, F. Jahnke, and T. O. Wehling, Two-dimensional heterojunctions from nonlocal manipulations of the interactions, Nano Lett. 16, 2322 (2016).

[7] A. Raja, A. Chaves, J. Yu, G. Arefe, H. M. Hill, A. F. Rigosi, T. C. Berkelbach, P. Nagler, C. Schüller, T. Korn, C. Nuckolls, J. Hone, L. E. Brus, T. F. Heinz, D. R. Reichman, and A. Chernikov, Coulomb engineering of the bandgap and excitons in two-dimensional materials, Nat. Commun. 8, 15251 (2017).

[8] M. Florian, M. Hartmann, A. Steinhoff, J. Klein, A. W. Holleitner, J. J. Finley, T. O. Wehling, M. Kaniber, and C. Gies, The dielectric impact of layer distances on exciton and trion binding energies in van der Waals heterostructures, Nano Lett. 18, 2725 (2018).

[9] R. Rosati, F. Lengers, D. E. Reiter, and T. Kuhn, Spatial control of carrier capture in two-dimensional materials: Beyond energy selection rules, Phys. Rev. B 98, 195411 (2018).

[10] D. Xiao, G.-B. Liu, W. Feng, X. Xu, and W. Yao, Coupled spin and valley physics in monolayers of MoS $\mathrm{S}_{2}$ and other group-vi dichalcogenides, Phys. Rev. Lett. 108, 196802 (2012).

[11] G.-B. Liu, W.-Y. Shan, Y. Yao, W. Yao, and D. Xiao, Three-band tight-binding model for monolayers of group-VIB transition metal dichalcogenides, Phys. Rev. B 88, 085433 (2013).

[12] M. Selig, G. Berghäuser, A. Raja, P. Nagler, C. Schüller, T. F. Heinz, T. Korn, A. Chernikov, E. Malic, and A. Knorr, Excitonic linewidth and coherence lifetime in monolayer transition metal dichalcogenides, Nat. Commun. 7, 13279 (2016). 
[13] S. Brem, A. Ekman, D. Christiansen, F. Katsch, M. Selig, C. Robert, X. Marie, B. Urbaszek, A. Knorr, and E. Malic, Phonon-assisted photoluminescence from indirect excitons in monolayers of transition-metal dichalcogenides, Nano Lett. 20, 2849 (2020).

[14] R. Rosati, K. Wagner, S. Brem, R. Perea-Causín, E. Wietek, J. Zipfel, J. D. Ziegler, M. Selig, T. Taniguchi, K. Watanabe, A. Knorr, A. Chernikov, and E. Malic, Temporal evolution of low-temperature phonon sidebands in transition metal dichalcogenides, ACS Photonics 7, 2756 (2020).

[15] R. Rosati, S. Brem, R. Perea-Causín, R. Schmidt, I. Niehues, S. M. de Vasconcellos, R. Bratschitsch, and E. Malic, Strain-dependent exciton diffusion in transition metal dichalcogenides, 2D Mater. 8, 015030 (2021).

[16] Z. Jin, X. Li, J. T. Mullen, and K. W. Kim, Intrinsic transport properties of electrons and holes in monolayer transitionmetal dichalcogenides, Phys. Rev. B 90, 045422 (2014).

[17] K. Wagner, J. Zipfel, R. Rosati, E. Wietek, J. D. Ziegler, S. Brem, R. Perea-Causín, T. Taniguchi, K. Watanabe, M. M. Glazov, E. Malic, and A. Chernikov, Nonclassical Exciton Diffusion in Monolayer WSe 2 , Phys. Rev. Lett. 127, 076801 (2021).

[18] D. Reiter, M. Glanemann, V. M. Axt, and T. Kuhn, Controlling the capture dynamics of traveling wave packets into a quantum dot, Phys. Rev. B 73, 125334 (2006).

[19] D. Reiter, M. Glanemann, V. M. Axt, and T. Kuhn, Spatiotemporal dynamics in optically excited quantum wire-dot systems: Capture, escape, and wave-front dynamics, Phys. Rev. B 75, 205327 (2007).

[20] R. Rosati, D. E. Reiter, and T. Kuhn, Lindblad approach to spatiotemporal quantum dynamics of phonon-induced carrier capture processes, Phys. Rev. B 95, 165302 (2017).

[21] F. Lengers, T. Kuhn, and D. E. Reiter, Phonon-mediated exciton capture in Mo-based transition metal dichalcogenides, Phys. Rev. Research 2, 043160 (2020).

[22] T. Sohier, M. Calandra, and F. Mauri, Two-dimensional Fröhlich interaction in transition-metal dichalcogenide monolayers: Theoretical modeling and first-principles calculations, Phys. Rev. B 94, 085415 (2016).

[23] P. Vasa, C. Ropers, R. Pomraenke, and C. Lienau, Ultra-fast nano-optics, Laser \& Photonics Reviews 3, 483 (2009).

[24] M. Reichelt and T. Meier, Shaping the spatiotemporal dynamics of the electron density in a hybrid metal-semiconductor nanostructure, Opt. Lett. 34, 2900 (2009).

[25] R. Rosati and F. Rossi, Microscopic modeling of scattering quantum non-locality in semiconductor nanostructures, Appl. Phys. Lett. 103, 113105 (2013).

[26] R. Rosati, F. Dolcini, and F. Rossi, Dispersionless propagation of electron wavepackets in single-walled carbon nanotubes, Appl. Phys. Lett. 106, 243101 (2015).

[27] G. Wang, A. Chernikov, M. M. Glazov, T. F. Heinz, X. Marie, T. Amand, and B. Urbaszek, Colloquium: Excitons in atomically thin transition metal dichalcogenides, Rev. Mod. Phys. 90, 021001 (2018).

[28] T. Mueller and E. Malic, Exciton physics and device application of two-dimensional transition metal dichalcogenide semiconductors, npj 2D Mater. Appl. 2, 29 (2018).

[29] M. Selig, G. Berghäuser, M. Richter, R. Bratschitsch, A. Knorr, and E. Malic, Dark and bright exciton formation, thermalization, and photoluminescence in monolayer transition metal dichalcogenides, 2D Mater. 5, 035017 (2018).

[30] S. Brem, M. Selig, G. Berghaeuser, and E. Malic, Exciton relaxation cascade in two-dimensional transition metal dichalcogenides, Sci. Rep. 8, 8238 (2018).

[31] F. Lengers, R. Rosati, T. Kuhn, and D. E. Reiter, Spatiotemporal dynamics of coulomb-correlated carriers in semiconductors, Phys. Rev. B 99, 155306 (2019).

[32] F. Rossi and T. Kuhn, Theory of ultrafast phenomena in photoexcited semiconductors, Rev. Mod. Phys. 74, 895 (2002).

[33] R. Rosati, R. C. Iotti, F. Dolcini, and F. Rossi, Derivation of nonlinear single-particle equations via many-body lindblad superoperators: A density-matrix approach, Phys. Rev. B 90, 125140 (2014). 